\title{
Representative spectrum of road traffic noise
}

\author{
Rufin Makarewicz ${ }^{\dagger}$ and Yasuyuki Sato \\ Department of Acoustic Design, Kyushu Institute of Design, \\ 9-1, Shiobaru 4-chome, Minami-ku, Fukuoka, 815 Japan
}

(Received 30 November 1995)

\begin{abstract}
The representative spectrum of traffic noise is expressed in terms of the normalized Aweighted sound pressure level, $\delta L_{n}$. Assuming that the traffic noise annoyance is measured by the time-average sound level, the dependence of $\delta L_{n}$ upon the traffic speed and the percentage of heavy vehicles has been derived. For traffic without heavy vehicles, an increase in traffic speed causes $\delta L_{n}$ to decrease at low frequencies. The presence of heavy vehicles destroys the dependence of $\delta L_{n}$ upon the traffic speed and may increase the value of $\delta L_{n}$ a few decibels at low frequencies.
\end{abstract}

Keywords: Noise barrier, Road traffic

PACS number: 43. 50. $-\mathrm{x}$

\section{INTRODUCTION}

In studying the problem of traffic noise generation, it is convenient to model a stream of cruising vehicles (i.e. lane of a road) as a single source. Particularly, the noise spectrum of such a source is useful for noise barrier investigation. ${ }^{1-4)}$ Most recently, a noise spectrum, based on measurements taken in various European countries and reviewed by the noise barrier standards committee (CENTC 226/WG6/TG1), has been introduced. ${ }^{5)}$ This spectrum, in octave bands, is shown in Fig. 1. Its definition can be given by,

$$
\delta L_{n}=L_{\mathrm{p} n}+\Delta L_{n}-L_{\mathrm{pA}},
$$

where $L_{\mathrm{p} n}$ expresses the sound pressure level in the $n$-th frequency band, $L_{\mathrm{pA}}$ denotes the A-weighted sound pressure level, and $\Delta L_{n}=-16,-9,-3,0$, $+1,+1$ are the A-weightings, taken for octave bands that are characterized by their central frequencies, $f_{n}=125,250,500,1,000,2,000$ and 4,000 Hz. The quantity defined by Eq. (1) is called the normalized A-weighted sound pressure level. In view of its definition and the definition of the

\footnotetext{
${ }^{\dagger}$ On leave from the Institute of Acoustics, A. Micki-
} ewicz University, Poznan (Poland)
A-weighted sound pressure level,

$$
L_{\mathrm{pA}}=10 \log \left\{\sum_{n} 10^{\left[L_{\mathrm{p} n}+\Delta L_{n}\right] / 10}\right\},
$$

the problem posed in this paper can be formulated as the following question: what are representative values of the sound pressure level of traffic noise, $L_{\mathrm{p} n}$ ? Road traffic consists of vehicles belonging to different types. It has been accepted to assume that there are either two or three main categories of vehicles, such as light, medium, and heavy vehicles. The noise generated by a vehicle on a road is a function of its operation mode and the road's surface. When the vehicle is cruising with steady speed $V$, both $L_{\mathrm{p} n}$ and $L_{\mathrm{pA}}$ are related to $V$.

Therefore, we expect the normalized A-weighted sound pressure spectrum, $\delta L_{n}$ (Eq. (1)), to be a function of traffic speed, $V$, as well. However, $L_{\mathrm{p} n}$ for a light and heavy vehicles are not the same (Fig. 2) : $L_{\mathrm{p} n}^{(1)} \neq L_{\mathrm{p} n}^{(2)}$.

Although it may be intuitive to use the arithmetic mean, $\left[L_{\mathrm{p} n}^{(1)}+L_{\mathrm{p} n}^{(2)}\right] / 2$, to get the representative sound pressure spectrum, $L_{\mathrm{p} n}$, we must use an alternate method, as the noise contributions due to light and heavy vehicles are not identical. It arises from the relative proportion (mix) of both categories, which depends on the day, the lane of a highway, etc. 


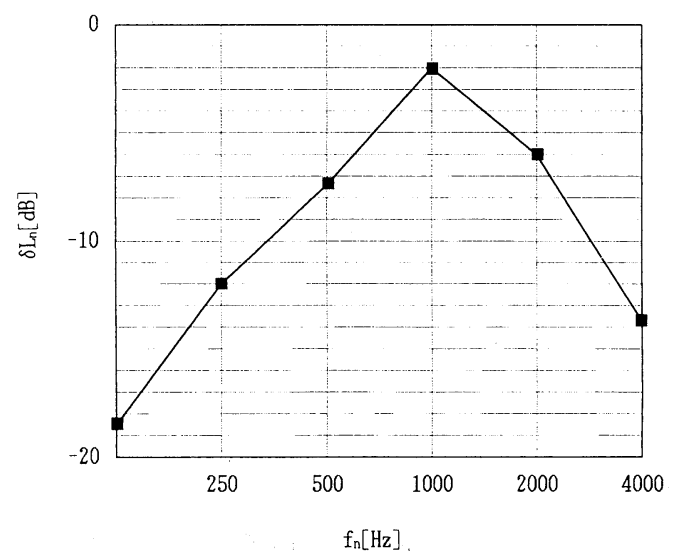

Fig. 1 The normalized A-weighted sound pressure spectrum for European traffic noise. ${ }^{5)}$

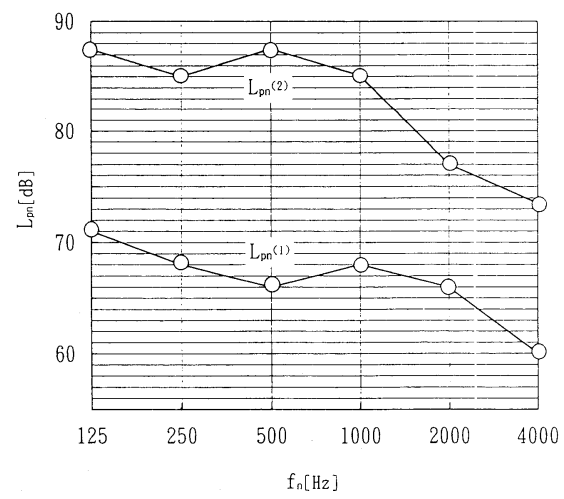

Fig. 2 Sound pressure level for a light and heavy vehicle, $L_{\mathrm{p} n}^{(1)}, L_{\mathrm{p} n}^{(2)}$, measured $1.2 \mathrm{~m}$ above the ground at a distance of $15 \mathrm{~m}$, while the speed of cruising is $V=88 \mathrm{~km} / \mathrm{h}^{6}{ }^{\text {) }}$

Moreover, the acoustic power of a light vehicle is considerably less than that of a heavy vehicle.

Before we show how to calculate the representative spectrum, we make use of the definition of the sound pressure level and rewrite Eq. (1) in the following form,

$$
\delta L_{n}=10 \log \left\{\frac{\left\langle p_{n \mathrm{~A}}{ }^{2}\right\rangle}{\sum_{n}\left\langle p_{n \mathrm{~A}}{ }^{2}\right\rangle}\right\},
$$

where

$$
\left\langle p_{n \mathrm{~A}}{ }^{2}\right\rangle=10^{\triangle L_{n} / 10}\left\langle p_{n}{ }^{2}\right\rangle .
$$

Here $\left\langle p_{n}{ }^{2}\right\rangle$ and $\left\langle p_{n \mathrm{~A}}{ }^{2}\right\rangle$ express the average square sound pressure in the $n$-th frequency band and the A-weighted average square sound pressure in the same frequency band, respectively. Now it becomes clear that the representative spectrum $\delta L_{n}$ (Eq. 1) is related to the average square sound pressure, $\left\langle P_{n}^{2}\right\rangle$. In the next section it will be shown that brackets $\langle>$ mean the time average.

\section{TIME-AVERAGE-MEAN-SQUARE SOUND PRESSURE}

Assume that our task is to place a noise barrier close to a road. To use the appropriate noise spectrum, $\delta L_{n}$, we have to consider all vehicles which pass the receiver during time period $T$ of the highest annoyance. Since the annoyance is measured by the time-average sound level, $L_{\text {AeqT }}$, the time period $T$ under consideration corresponds to the maximum of $L_{\text {AeqT }}$ (e.g. 8:00-9:00, rush hour traffic). Now, have a look at the definition of the time average sound level :

$$
L_{\text {AeqT }}=10 \log \left\{\frac{\left\langle p_{\mathrm{A}}^{2}\right\rangle}{p_{0}^{2}}\right\}
$$

where

$$
\left\langle p_{\mathrm{A}}{ }^{2}\right\rangle=\frac{1}{T} \int_{0}^{T} p_{\mathrm{A}}^{2}(t) d t
$$

is the time-average-mean-square A-weighted sound pressure. It can be expressed as a sum of Aweighted average square sound pressures,

$$
\left\langle p_{n \mathrm{~A}}{ }^{2}\right\rangle=\frac{10^{\Delta L_{n} / 10}}{T} \int_{0}^{T} p_{n}{ }^{2}(t) d t,
$$

which have already appeared in the definition of $\delta L_{n}$ (Eq. (3)).

To capture the intuition of a noise event, assume the noise is emitted by a stream of identical vehicles that pass the receiver with the same velocity, such that the time variation of $p_{n}{ }^{2}(t)$ is as in Fig. 3 . Because the pass-by time, $\tau$, is much shorter than the averaging time $T$, we can write,

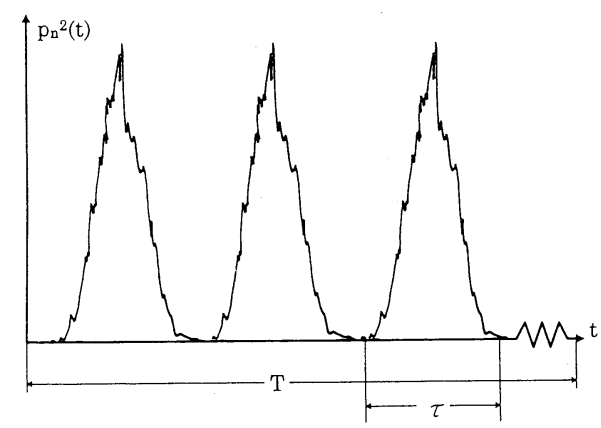

Fig. 3 Time variations of the square instantaneous sound pressure in the $n$-th frequency band for a stream of identical vehicles, which move with the same velocity. 


$$
\left\langle p_{n \mathrm{~A}}{ }^{2}\right\rangle=N \frac{10^{\Delta L n / 10}}{T} E_{n},
$$

where $N$ is the number of vehicles passing the receiver during the time $T$, and the integral

$$
E_{n}=\int_{-\infty}^{\infty} p_{n}^{2}(t) d t
$$

determines the sound exposure in the $n$-th frequency band. To be more realistic, suppose the noise has been produced by $N$ real vehicles of the same category, either light or heavy. In such a case we obtain,

$$
\left\langle p_{n \mathrm{~A}}{ }^{2}\right\rangle=N \frac{10^{\Delta L_{n} / 10}}{T} \tilde{E}_{n},
$$

where $\tilde{E}_{n}$ denotes the average over $N$ vehicles, which have gone past the receiver during the time period $T$. Considering $E_{n}^{(1)}, E_{n}^{(2)}, \cdots, E_{n}^{(N)}$ as a family of possible outcomes of the measurement, we apply the arithmetic average,

$$
\widetilde{E}_{n}=\frac{1}{N} \sum_{j}^{N} E_{n}^{(j)} .
$$

In reality the set of actual vehicles passing a given location differ from day to day. Thus we expect variations of $N$ and $\tilde{E}_{n} \cdot{ }^{7} \quad$ To get the representative value of $\left\langle p_{n \mathrm{~A}}{ }^{2}\right\rangle$ and finally $\delta L_{n}$ (Eq. (3)), we substitute the mean (over a few days) number of vehicles, $\bar{N}$, and the mean (over a few hundreds vehicles) sound exposure, $\overline{E_{n}}$, into Eq. (10).

For a stream of real vehicles that belong to two categories, the generalized form of Eq. (10) is,

$$
\left\langle p_{n \mathrm{~A}}{ }^{2}\right\rangle=\bar{N} \frac{10^{\Delta L_{n} / 10}}{T}\left[(1-k) \cdot \overline{E_{n 1}}+k \cdot \overline{E_{n 2}}\right],
$$

where $\bar{N} / T[\mathrm{veh} / \mathrm{s}]$ is the average flow rate of all vehicles (both light and heavy), and $k$ is the average percentage of heavy vehicles. In the above expression $\overline{E_{n 1}}$ and $\overline{E_{n 2}}$ denote the mean sound exposure in the $n$-th frequency band for light and heavy vehicles, respectively. Substitution of Eq. (12) into Eq. (3) yields the representative normalized A-weighted sound pressure level (Eq. (1)),

$$
\delta L_{n}=10 \log \left\{\frac{10^{\Delta L_{n / 10}}\left[(1-k) \cdot \overline{E_{n 1}}+k \cdot \overline{E_{n 2}}\right]}{\sum_{n} 10^{\Delta L_{n / 1}}\left[(1-k) \cdot \overline{E_{n 1}}+k \cdot \overline{E_{n 2}}\right]}\right\} .
$$

Note, that the average flow rate, $\bar{N} / T$, has dropped out and the average percentage of heavy vehicles, $k$, remains as a single traffic characteristic during the most annoying time period $T$ (e.g. $8: 00$ 9 : 00 a.m.).

\section{SOUND EXPOSURE}

To calculate the sound exposure in the $n$-th frequency band, $E_{n}(\mathrm{Eq} .(9))$, we have to know the time history of the square sound pressure in the corresponding frequency band, $p_{n}{ }^{2}(t)$. In free space, a cruising vehicle can be modeled by a nondirectional point source, which produces the spherical wave, ${ }^{8)}$

$$
p_{n}^{2}=\frac{P_{n} \rho C}{4 \pi r^{2}} .
$$

Here $P_{n}$ is the sound power in the $n$-th frequency band, $\rho c$ is the characteristic impedance of air, and $r$ is the distance between source and receiver. Above the ground in the Earth's atmosphere, the calculation of $p_{n}{ }^{2}(t)$ becomes more complex. The geometrical divergence given by Eq. (14) is disturbed by,

- ground effect,

- air absorption,

- scattering due to atmospheric turbulence,

- refraction caused by wind speed and temperature gradients.

Close to the source, the ground effect is the most important wave phenomenon,

$$
p_{n}{ }^{2}=\frac{P_{n} \rho c}{4 \pi r^{2}} \cdot G_{n}\left[d, H_{\mathrm{s}}, H_{0}, \sigma\right],
$$

where the ground factor $G_{n}{ }^{9-11)}$ is a function of the horizontal distance $d$, and heights of the source, $H_{\mathrm{s}}$, and the receiver, $H_{0}$ (Fig. 4). Flow resistivity, $\sigma$, characterizes the ground covering. While a vehicle is moving, the time variations of both distances, $r(t)$ and $d(t)$, occur. Thus, Eq. (9) and Eq. (15) combine into,

$$
E_{n}=\frac{P_{n} \rho C}{4 s_{0}} \cdot J_{n}
$$

where $s_{0}=1 \mathrm{~m}^{2}$ and the propagation factor, $J_{n}$, is given by an infinite integral,

$$
J_{n}=\frac{s_{0}}{\pi} \int_{-\infty}^{\infty} \frac{G_{n}\left[d(t), H_{\mathrm{s}}, H_{0}, \sigma\right]}{r^{2}(t)} d t .
$$

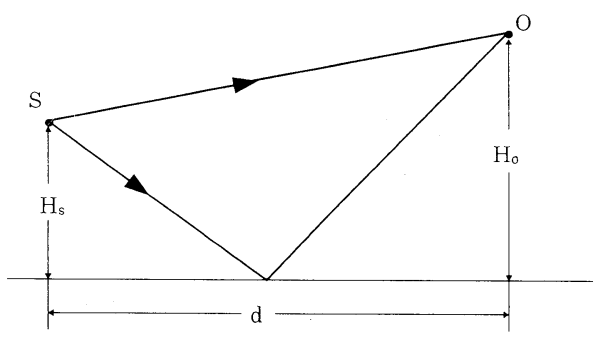

Fig. 4 The direct and reflected ray paths going from the source, $\mathrm{S}$, to the receiver, $\mathrm{O}$. 

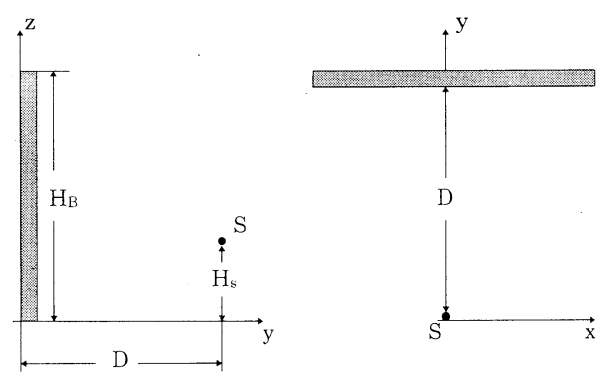

Fig. 5 The vehicle $S$ is moving with the speed $V$ along the barrier at the perpendicular distance $D$.

Assuming the vehicle location and ground properties are strictly determined, the average sound exposure can be calculated from,

$$
\overline{E_{n}}=\frac{\overline{P_{n}} \rho C}{4 s_{0}} \cdot J_{n},
$$

where $\overline{P_{n}}$ means the average sound power in the $n$-th frequency band. For the purpose of noise barrier design, we consider vehicles which cruise with a steady speed, $V$, along the barrier at the perpendicular distance $D$ (Fig. 5). Considering the height of the barrier as receiver's height, $H_{0}=H_{\mathrm{B}}$, we get the explicit dependence of the distance, $r$, upon the time,

$$
r=\left\{(V t)^{2}+D^{2}+\left(H_{\mathrm{s}}-H_{\mathrm{o}}\right)^{2}\right\}^{1 / 2} .
$$

Now, assuming that the height of a vehicle $\left(H_{\mathrm{s}}\right)$ is known, we are able to calculate the propagation factor, $J_{n}$, for any frequency band (Eqs. (17), (19)). Since the height of a light vehicle is different from that of a heavy vehicle, $H_{\mathrm{s} 1} \neq H_{\mathrm{s} 2}$, we can expect two different values of the propagation factor, $J_{n 1}$ and $J_{n 2}$, for the same frequency band.

To obtain a first approximation to $J_{n}$, we set $H_{\mathrm{s} 1}=H_{\mathrm{s} 2}=0$ for both categories of vehicles and assume that the direct and reflected waves do not interfere with each other: $G_{n}=2$. Thus, we arrive at the simplified form of the propagation factor (Eqs. (17), (19)),

$$
J=\frac{2 s_{0}}{V \sqrt{D^{2}+H_{0}^{2}}},
$$

which is the same for each frequency band. Equalities, $J_{1}=J_{2}=\cdots=J_{n}$, mean that the ground effect does not change the shape of the noise spectrum. However, for $H_{\mathrm{s}}>0$ it is no longer true.

\section{SOUND POWER OF A VEHICLE}

In this section we consider the average sound power, $P_{n}$, which is necessary for $E_{n}$ (Eq. (18)) and ultimately for $\delta L_{n}$ (Eq. (13)) calculation.

Yoshihisa et al ${ }^{12)}$ have measured time average sound level, $L_{\mathrm{AT} n}$, of traffic noise and then calculated the sound power, $P_{n}$, for different velocities $V$. Figure 6 shows an example of their results for light

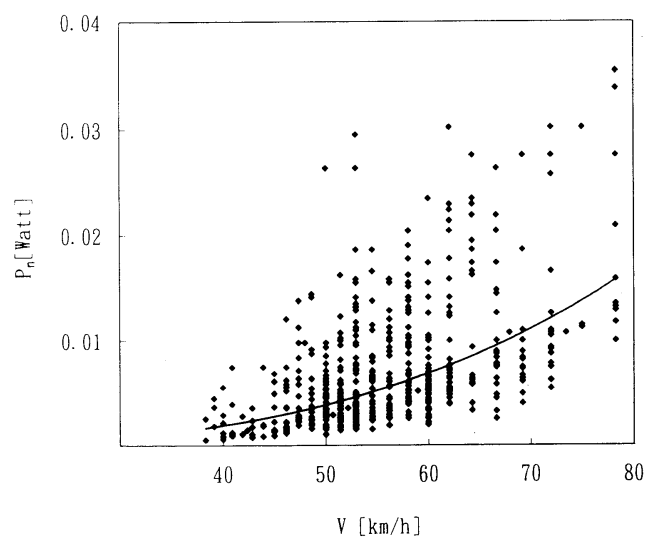

Fig. 6 The sound power of noise, $P_{n}$, for the octave frequency band $f_{n}=1,000 \mathrm{~Hz}$, which is generated by a light vehicle $\left(A_{n 1}=1.6 \cdot 10^{-8}\right.$, $m n 1=3.2$, see Table 1).

\begin{tabular}{|c|c|c|c|c|}
\hline \multirow{2}{*}{$\begin{array}{l}\text { Frequency band } \\
f_{n}(\mathrm{~Hz})\end{array}$} & \multicolumn{2}{|c|}{ Light vehicles } & \multicolumn{2}{|c|}{ Heavy vehicles } \\
\hline & $A_{n 1}$ & $m n 1$ & $A_{n 2}$ & $m n 2$ \\
\hline 125 & $3.31 \cdot 10^{-5}$ & 1.05 & $5.93 \cdot 10^{-5}$ & 1.68 \\
\hline 250 & $1.48 \cdot 10^{-5}$ & 1.32 & $1.47 \cdot 10^{-7}$ & 3.24 \\
\hline 500 & $4.13 \cdot 10^{-7}$ & 2.25 & $2.71 \cdot 10^{-7}$ & 2.96 \\
\hline 1,000 & $1.64 \cdot 10^{-8}$ & 3.16 & $1.65 \cdot 10^{-8}$ & 3.60 \\
\hline 2,000 & $4.08 \cdot 10^{-9}$ & 3.35 & $7.40 \cdot 10^{-9}$ & 3.58 \\
\hline 4,000 & $1.28 \cdot 10^{-8}$ & 2.63 & $2.32 \cdot 10^{-8}$ & 2.88 \\
\hline
\end{tabular}

Table 1 Coefficients $A$ and $m$, which determine the average sound power, $\overline{P_{n}}$, for light and heavy vehicles (Eqs. 21). 
vehicles and $f_{n}=1,000 \mathrm{~Hz}$. Regression analysis for 704 light vehicles yields,

$$
\overline{P_{1}}=1.64 \cdot 10^{-8} \cdot(\bar{V})^{3.16},
$$

where $\bar{V}$ expresses the average speed in $\mathrm{km} / \mathrm{h}$. For heavy vehicles and the same frequency band $f_{n}=$ $1,000 \mathrm{~Hz}$, we get

$$
\overline{P_{2}}=1.65 \cdot 10^{-8} \cdot(\bar{V})^{3.60} .
$$

So, for any frequency band $(n)$ we can write,

$$
\overline{P_{n 1}}=A_{n 1} \cdot \bar{V}^{m n 1}, \overline{P_{n 2}}=A_{n 2} \cdot \bar{V}^{m n 2} .
$$

Table 1 contains the complete set of coefficients $A_{n 1}$, $m n 1$ and $A_{n 2}, m n 2$, for both categories of vehicles. Equations (21) are used for the description of vehicle sound power in many countries.

\section{NORMALIZED A-WEIGHTED SOUND PRESSURE SPECTRUM}

In the most general case, when the ground effect modifies the noise spectrum and Eq. (17) yields different values of $J_{n 1}\left(H_{\mathrm{S} 1}, \cdots\right)$ and $J_{n 2}\left(H_{\mathrm{S} 2}, \cdots\right)$ for different frequency bands, the normalized Aweighted sound pressure spectrum can be calculated from (Eqs. (13), (18)),

$\delta L_{n}=10 \log \left\{\frac{10^{\Delta L_{n} / 10}\left[(1-k) \cdot \overline{P_{n 1}} \cdot J_{n 1}+k \cdot \overline{P_{n 2}} \cdot J_{n 2}\right]}{\sum_{n} 10^{\Delta L_{n} / 10}\left[(1-k) \cdot \overline{P_{n 1}} \cdot J_{n 1}+k \cdot \overline{P_{n 2}} \cdot J_{n 2}\right]}\right\}$.

Assuming $H_{\mathrm{s} 1}=H_{\mathrm{s} 2}=0$ and a lack of coherence between the direct and reflected waves, Eq. (17) gives : $J_{n 1}=J_{n 2}=J$ (Eq. 20). In such a case, expression (22) simplifies to the form,

$$
\delta L_{n}=10 \log \left\{\frac{10^{\Delta L_{n} / 10}\left[(1-k) \cdot \overline{P_{n 1}}+k \cdot \overline{P_{n 2}}\right]}{\sum_{n} 10^{\Delta L_{n} / 10}\left[(1-k) \cdot \overline{P_{n 1}}+k \cdot \overline{P_{n 2}}\right]}\right\} .
$$

Finally, when a stream of vehicles is moving with a steady speed, $V$, by substitution of Eqs. (21) into Eq. (23) we obtain,

$$
\begin{aligned}
& \delta L_{n}=10 \\
& \cdot \log \left\{\frac{10^{\Delta L_{n / 10}}\left[(1-k) \cdot A_{n 1} \cdot \bar{V}^{m n 1}+k \cdot A_{n 2} \cdot \bar{V}^{m n 2}\right]}{\sum_{n} 10^{\Delta L_{n} / 10}\left[(1-k) \cdot A_{n 1} \cdot \bar{V}^{m n 1}+k \cdot A_{n 2} \cdot \bar{V}^{m n 2}\right]}\right\},
\end{aligned}
$$

where coefficients $A$ and $m$ (for Japanese roads) are given in the Table 1.

In a particular case of road traffic, which consists only from light vehicles, $k=0$, Eq. (24) takes the form,

$$
\delta L_{n}=10 \log \left\{\frac{10^{\Delta L_{n} / 10} \cdot A_{n 1} \cdot \bar{V}^{m n 1}}{\sum_{n} 10^{\Delta L_{n / 10}} \cdot A_{n 1} \cdot \bar{V}^{m n 1}}\right\} .
$$

In all above equations, the traffic velocity, $\bar{V}$, is expressed in kilometers per hour.

\section{NOISE SPECTRA OF TRAFFIC FROM JAPANESE ROADS}

Let's assume that vehicles of both categories are moving with the same speed, $V$. Figure 7 shows

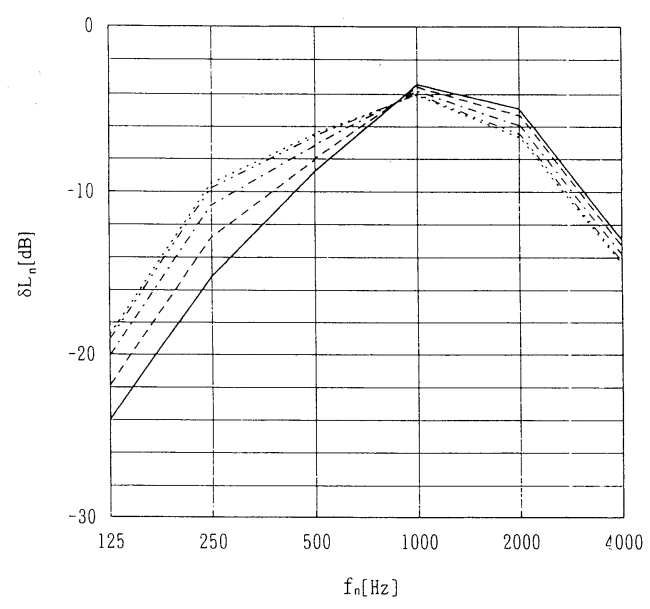

Fig. 7 The normalized A-weighted sound pressure spectrum (Eqs. (1), (24)) for traffic speed $V=60 \mathrm{~km} / \mathrm{h}$ and $k=0$ (-), $0.05(---)$, $0.2(-\cdot \cdot), 0.6(-\cdots)$, and $1.0(\cdots)$.

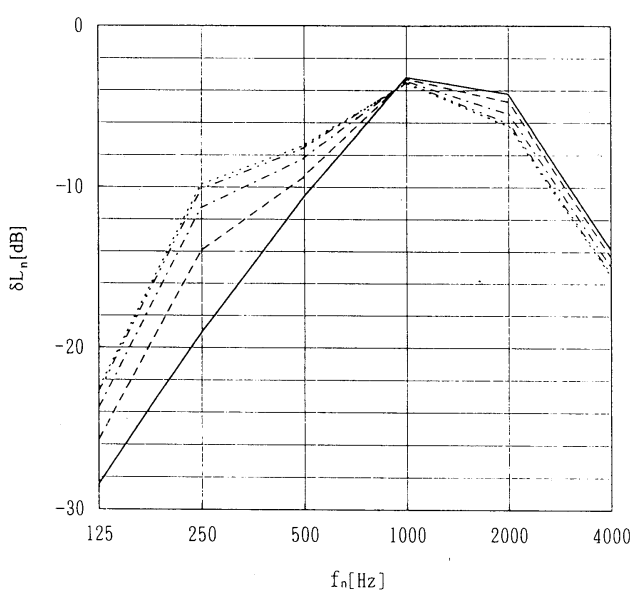

Fig. 8 The normalized A-weighted sound pressure spectrum (Eqs. (1), (24)) for traffic speed $V=100 \mathrm{~km} / \mathrm{h}$ and $k=0(-), 0.05(---)$, $0.2(-\cdot \cdot), 0.6(-\cdot-)$, and $1.0(\cdots)$. 
the changes of the normalized A-weighted sound pressure spectrum, $\delta L_{n}$ (Eq. (24)), when the percentage of heavy vehicles increases from $k=0$ to $k=1$, while $V=60 \mathrm{~km} / \mathrm{h}$. The values of $\delta L_{n}$ for low frequencies $(f<500 \mathrm{~Hz})$ increase with increasing $k$. In Fig. 8 the variations of $\delta L_{n}$ with $k$ are presented, while the speed $V=100 \mathrm{~km} / \mathrm{h}$. In the latter case the influence of heavy vehicles on $\delta L_{n}$ is more distinct.

\section{CONCLUSIONS}

Equation (24) makes it possible to calculate the representative spectrum of traffic noise, i.e., the normalized A-weighted sound pressure level, $\delta L_{n}$ (Eq. (1)), when the average traffic speed $\bar{V}(\mathrm{~km} / \mathrm{h})$ and the percentage of heavy vehicles, $0 \leq k \leq 1$, are known. Our results indicate that both quantities, i.e. $\bar{V}$ and $k$, play an important role, especially at frequencies less than $500 \mathrm{~Hz}$ (Figs. 7, 8).

Inspection of Fig. 1 and Fig. 8 leads to the conclusion that the representative spectrum of noise traffic for European roads is almost identical with that for Japanese roads, when the traffic speed equals $V=60$ $\mathrm{km} / \mathrm{h}$ and the percentage of heavy vehicles is $k=0.2$.

\section{ACKNOWLEDGEMENTS}

The authors would like to thank Dr. K. Yoshihisa (Meijo University) and Prof. Dr. H. Tachibana (University of Tokyo) for making the sound power data available.

\section{REFERENCES}

1) D. H. Crombie, D. C. Hothersall, and S. N. Chandler,
"Wilde, multiple-edge noise barriers," Appl. Acoust. 44, 353-364 (1995).

2) K. Fujiwara and T. Ohkubo, "Sound shielding efficiency of a noise barrier with soft surface and soft round obstacle at the edge," ICA-95, 97-100 (1995).

3) K. R. Fyfe, C. C. Harrison, and L. Cremers, "Performance of barriers and berms for road noise attenuation," ICA-95, 101-104 (1995).

4). R. J. Alfredson and X. Du, "Special shapes and treatments for noise barriers," Inter-Noise 95, 381-384 (1995).

5) G. R. Watts, D. H. Crombie, and D. C. Hothersall, "Acoustic performance of new designs of traffic noise barriers : full scale tests," J. Sound Vib. 177, 289-305 (1994).

6) W. Bowlby, "Highway noise prediction and control," in Acoustical Measurements and Noise Control, C. M. Harris, Ed. (McGraw- Hill, New York, 1991).

7) R. Makarewicz, "Statistical properties of equivalent continuous A-weighted sound pressure level," J. Acoust. Soc. Jpn. (E) 14, 25-28 (1992).

8) B. Favre, "Factors affecting traffic noise and method of prediction," in Transportation Noise Reference Book, P. M. Nelson, Ed. (Butterworths, London, 1987).

9) K. Attenborough, "Review of ground effects on outdoor sound propagation from continuous broadband source," Appl. Acoust. 24, 289-319 (1988).

10) T. Kawai, "On Sound propagation above a locally reacting boundary," J. Acoust. Soc. Jpn. (J) 39, 374379 (1983).

11) Y. Miki, "Acoustical properties of porous materials -Modifications of Delany-Bazley models," J. Acoust. Soc. Jpn. (E) 11, 19-24 (1990).

12) K. Yoshihisa, K. Tatsuda, and H. Tachibana, "Sound power levels of road vehicles measured by the squareintegrating technique," Inter-Noise 91, 353-356 (1991). 